\title{
Assessing compliance with healthy eating recommendations for food groups: comparison of a food frequency questionnaire and diet diary
}

\author{
S. Whybrow ${ }^{1}$, L. Craig ${ }^{2}$, H. Clark ${ }^{2}$ and G. McNeill ${ }^{2}$ \\ ${ }^{1}$ Rowett Institute of Nutrition and Health and ${ }^{2}$ Public Health Nutrition Research Group, University of Aberdeen, \\ Aberdeen AB25 2ZD
}

The Eatwell plate was developed to give a food-based visual representation of the balance of five food groups for a recommended healthy diet. The five groups being: bread, rice, potatoes, pasta and other starchy foods (starchy), fruit and vegetables (F\&V), milk and dairy foods (dairy), meat, fish, eggs, beans and other non-dairy sources of protein (protein) and foods and drinks high in fat or sugar (HFHS) ${ }^{(1)}$. Manufactured dishes comprise ingredients from several food groups and consumers must consider how these fit with the proportions of the Eatwell plate. This will present a difficulty for many consumers, and is a further level of complexity in estimating how well a diet compares to the recommendations. A simple to use tool that is not reliant on the consumers' nutritional knowledge could be beneficial in providing them with information on their Eatwell plate proportions, and could be used in research to monitor the progress of the population's diet towards health eating goals. The aim of the study was to assess the relative validity of the Scottish Collaborative Group Food Frequency Questionnaire (FFQ) ${ }^{(2)}$, compared to food intake diaries, in estimating the Eatwell plate proportions of the diets of Scottish adults.

Dietary data were taken from two existing studies when subjects were consuming their normal diets ${ }^{(3,4)}$. Subjects $(110 \mathrm{~F}$ and $94 \mathrm{M})$ completed an FFQ followed by a weighed food intake record (WR) for 4, 6 or 7 days. Proportions of the five Eatwell food groups were estimated for each of the foods and beverages recorded by subjects in their WR, and for all items $(n=155)$ of the FFQ. Composite foods were disaggregated where necessary. Weights of milk and fruit juices were halved to avoid their high water content having a large effect on the Eatwell plate proportions. Eatwell plate proportions for each subject's overall diet were calculated for the FFQ and WR. Subjects were grouped into thirds of intake for each Eatwell food group and dietary method, and the percentages of subjects in the same third and opposite third by the two methods calculated. The weighted kappa statistic was calculated for each of the Eatwell food groups to assess the agreement between the FFQ and WR.

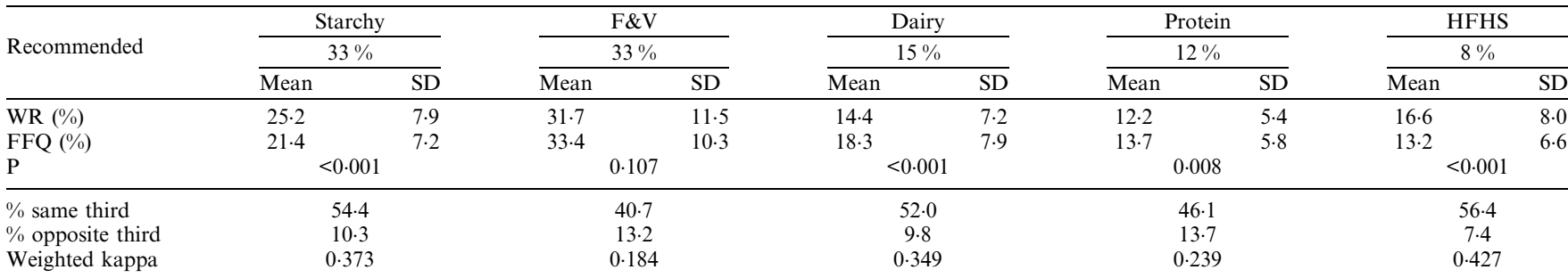

Significantly more food was reported using the FFQ than the WR (1646 (596) and 1393 (389) g/d respectively, p < 0.001), and there were significant differences in the Eatwell proportions between the two methods. Overall the classification of Eatwell proportions by the FFQ agreed (same third of intake) with the WR in $50 \%$ of the cases, and disagreed (opposite third of intake) in $11 \%$ of cases. The overall agreement was fair $(\mathrm{Kw}=0 \cdot 314)$. Part of the difference in agreement could be due to within subject variation in food intake (the FFQ and WR were not completed concurrently) and because FFQs and WRs do not measure exactly the same aspects of the diet.

Scottish Collaborative Group Food Frequency Questionnaire gave fair agreement with weighed food diaries in estimating the Eatwell plate proportions at the group level, making it a suitable tool for estimating this aspect of overall diet quality in groups of subjects. Caution is needed when estimating the Eatwell plate proportions of individuals' diets, however.

1. Food Standards Agency (2010) The eatwell plate.

2. Masson L, McNeill G, Tomany J et al. (2003) Public Health Nutrition 6, 313-321. www.foodfrequency.org.

3. Stubbs R, O'Reilly L, Whybrow S et al. (2014) British Journal of Nutrition 111, 2032-2043.

4. Whybrow S, Mayer C, Kirk T et al. (2007) Obesity Research 15, 673-685. 